\title{
Classical nova V339 Del (Nova Del 2013) - a short review
}

\author{
Drahomír Chochol $^{* 1}$, Sergey Shugarov ${ }^{1,2}$, Nataly Katysheva ${ }^{2}$ and Igor Volkov ${ }^{2}$ \\ ${ }^{1}$ Astronomical Institute of the Slovak Academy of Sciences, 05960 Tatranská Lomnica, Slovakia \\ ${ }^{2}$ Sternberg Astronomical Institute of the Moscow State University, 119991 Moscow, Russia \\ E-mail: checholdta3.sk, Shugarovdta3.sk, natkat2006amail.ru, \\ hwpdyandex.ru
}

Multifrequency observations of the classical CO nova V339 Del, detected in all frequencies from $\gamma$-rays to radio, are reviewed. Nova V339 Del was discovered on 2013 August 14.584 UT. The times of decline from the brightness maximum $V=4.4 \mathrm{mag}$, reached on 2013 August 16.47, UT, was estimated as $t_{2, V}=10$ days, $t_{3, V}=18$ days, so V339 Del can be classified as a fast nova with super-Eddington luminosity at maximum. The maximum-magnitude-rate-of-decline relations were used to determine an absolute magnitude at maximum $M_{V, \max }=-8.70 \pm 0.03$, $M_{B, \max }=-8.45 \pm 0.08$ and distance $d=3.2 \pm 0.3 \mathrm{kpc}$, using the interstellar extinction $E(B-V)=$ $0.184 \pm 0.035$. The distance to the nova found by different methods is in the range $2.7-4.54 \mathrm{kpc}$. The white dwarf mass was estimated from $M_{B, \max }$ as $M_{w d}=1.04 \pm 0.02 \mathrm{M}_{\odot}$. We suggest that the eruption occurred on the surface of a CO white dwarf. V339 Del is the first nova that has been observed to synthesize the element lithium. The dust consisted of amorphous carbon grains was detected in infrared region. The $U B V R_{C} I_{C}$ light curves of nova constructed from daily means of all available data, including our own observations obtained from its discovery till August 2015, are presented and used to show the track of the nova in the color-color diagram during the first 100 days. The dust particles formed in the dense clumps of ejecta were responsible for the $U$ light curve variability. Our medium-resolution spectrum of V339 Del taken in August 2015 by the 6m SAO telescope in North Caucasus confirms a non-spherical structure of the ejected shell.

The Golden Age of Cataclysmic Variables and Related Objects - III, Golden2015

7-12 September 2015

Palermo, Italy

${ }^{*}$ Speaker. 


\section{Introduction}

Classical novae are cataclysmic variables with outburst amplitude 6-19 mag, caused by thermonuclear runaway events on a surface of a white dwarf. The upper limit of the outburst amplitude was reached by Nova Cygni $1975=$ V1500 Cyg [48]. Classical novae arise in close binaries with orbital periods of a few hours, consisting of a red dwarf filling up its Roche-lobe and mass-accreting white dwarf. After the outburst, the photosphere of the white dwarf component of the nova expands to supergiant dimensions and engulfs the binary. Due to the strong wind a large part of the envelope is ejected and the photospheric radius shrinks.

Classical novae can be divided according to their photometric and spectroscopic appearance into the fast and slow. The classification is usually based on the time interval in which a given nova fades by 2 or 3 magnitudes $\left(t_{2}, t_{3}\right)$ below its maximum brightness. According to Downes and Duerbeck [1], the fast $\left(t_{2}<13, t_{3}<30\right.$ days) super-Eddington novae have smooth light curves with well defined maxima. They may be classified as He/N, "hybrid" Fe II or Fe II novae. The slow $\left(t_{2}>13, t_{3}>30\right.$ days) Eddington novae have often structured light curves and many of them have standstills at maximum and dust formation at later stages. They belong to the Fe II spectroscopic type.

\section{Basic characteristics of the nova}

Nova Delphini 2013 was discovered by K. Itagaki [2] on 2013 August 14.584 UT at mag 6.8 at the coordinates $\alpha_{2000}=20^{h} 23^{m} 30^{s} .73, \delta_{2000}=20^{\circ} 46^{\prime} 04^{\prime}$. 1 . The variable, preliminary designated as PNV J20233073+2046041, was named V339 Del [3]. The first spectra of the nova on August 14.909 UT [4] and August 14.87 UT [5] enabled to classify it as Fe II type CO classical nova. The high resolution spectra of the nova, taken between Aug. 14.84 - 16.86 [6] provided the equivalent width of $\mathrm{Na}$ I line as $0.3945(30) \AA$, which corresponds to a reddening $E(B-V)=0.182$, following the calibration by [7].

In September 2013 Chochol et al. [8] presented $B, V$ photometry and spectroscopy of the nova V339 Del at the international workshop in Tatranská Lomnica, Slovakia. They found that nova reached brightness maximum $V_{\max }=4.4$ and $B_{\max }=4.76$ on 2013 August 16.47 (JD 2456520.97, day 0 ) and estimated the times of decline from brightness maximum to be $t_{2, V}=10$ days, $t_{3, V}=$ 18 days, which allowed to classify V339 Del as a fast super-Eddington nova. Various maximummagnitude-rate-of-decline (MMRD) relations [1] enabled [8] to determine the absolute magnitude at maximum $M_{V, \max }=-8.70 \pm 0.03, M B_{\max }=-8.45 \pm 0.08$ and the distance $d=3.2 \pm 0.3 \mathrm{kpc}$, using the interstellar extinction in agreement with the value found by [6]. The mass of the white dwarf was estimated from the $M B_{\max }$ and the well known formula [9] as $M_{w d}=1.04 \pm 0.02 \mathrm{M}_{\odot}$. Burlak et al. [10] estimated the interstellar reddening $E(B-V)=0.18$ using maps of Galactic extinction and the MMRD relations [1] found that the distance to the nova is $2.7-3 \mathrm{kpc}$. Schaefer et al. [11] reported near-infrared interferometric observations of V339 Del with the CHARA Array at Mt. Wilson, which started a day after discovery and lasted during the first 43 days of the outburst. They detected an ellipticity in the light distribution, suggesting a prolate or bipolar structure that develops as early as the second day of the outburst. Combining the angular expansion rate with the outflow speed near the continuum forming layer $V_{\text {ejection }}=613 \pm 79 \mathrm{~km} / \mathrm{s}$ (based on an analysis 
of spectra from the archive of the Astronomical Ring for Access to Spectroscopy (ARAS)), the above authors derived a geometric distance to the nova of $4.54 \pm 0.59 \mathrm{kpc}$. The true distance of the nova depends on geometry of the expanding shell and the inclination angle of the nova binary orbit. Shore [12] estimated that the distance to V339 Del is $4.2 \mathrm{kpc}$ comparing its UV spectra with those of the CO nova OS And.

The nova progenitor was found by [13] as the blue star USNO-B1 1107-0509795 ( $B=17.2$ $17.4 \mathrm{mag}$ ), so the total outburst amplitude was $\sim 12.6 \mathrm{mag}$ in $B$. The increase of brightness was very fast, because the nova was in quiescence (17.1 mag) 14 hours before its discovery. Munari and Henden [14] found the progenitor also on the Asiago 1979-82 plates: $B=17.27, V=17.60$ and in AAVSO Photometric All-Sky Survey (APASS) observations in 2012: $B=17.33$. The amplitude of the variations in $B$ was 0.9 mag with color as expected for progenitor dominated by emissions from an accretion disk. Deacon et al. [15] found observations of progenitor in the PanSTARRS 1 image archive. The data were obtained in the last 1.2 years before the nova outburst, when the nova's variability was below the upper photometric limit of the archive plate photometry [14].

\section{Multifrequency observations of V339 Del and results}

\subsection{Gamma-rays data}

V339 Del like three other classical novae V959 Mon, V1324 Sco and V1369 Cen was detected in 2012-13 by Fermi-Lat collaboration [16,17] as soft spectrum transient $\gamma$-ray sources, over 2-3 weeks duration. The Fermi-LAT high energy $\gamma$-ray telescope, covering the energy range $20 \mathrm{MeV}$ $300 \mathrm{GeV}$, has been continuously scanning the sky from its launch in 2008 , so it is suitable to detect transient sources. In V339 Del the maximum of $\gamma$-ray flux followed the optical maximum by 6 days. The ejected material initially blocked the high-energy photons. The gamma rays photons are absorbed via photon-atom interactions. $100 \mathrm{MeV}$ emission is detected later, when the density due to the expansion of the shell drops and the ejecta become transparent for $\gamma$-rays. They are produced by collisions between relativistic protons with the nova ejecta (hadronic scenario) or Inverse Compton/bremsstrahlung emission from relativistic electrons (leptonic scenario). According to [18], the measured ratio of $\gamma$-ray and optical luminosities sets a lower limit on the fraction of the shock power used to accelerate relativistic particles, which is in favour of hadronic scenario. The strong shocks needed to accelerate particles at relativistic energies develop within a mixed and delayed torus-bipolar expansion pattern for the ejecta [19].

\subsection{X-rays data}

V339 Del was regularly monitored by the X-ray telescope on board of the Swift multi-wavelength observatory and occasionally by the Chandra and XMM satellites. The first detection of an X-ray source in the hard 1-10 keV range of an X-rays on day 33 after maximum (AM) [20], was consistent with shocked gas in an expanding nova shell with no evidence for super-soft emission. Soft $\mathrm{X}$-rays in the range $0.3-1 \mathrm{keV}$ were first detected by [21] on day $58 \mathrm{AM}$ on very low flux level 0.0067 count/s [22]. On day $69 \mathrm{AM}$ the flux level increased to $1.5 \mathrm{count} / \mathrm{s}$, exclusively below 0.7 $\mathrm{keV}$, suggesting the unveiling of the hot WD atmosphere (start of the super-soft X-ray stage). According to [23] SSS reached maximum $\sim 100$ counts/s on day $86 \mathrm{AM}$. The rising count rate till day 
83 AM was interspersed by a few large dips. Large amplitude variations can be explained either by clumps in the ejecta, or WD photosphere temperature variations. QPO with a periodicity of $54 \mathrm{~s}$, later confirmed by Newton-XMM X-ray observations for day 97 AM [24], were also present in the data and interpreted by [25] as non radial g-mode pulsations of the WD. The high resolution X-ray spectrum (22-44 $\AA$ range) taken by the Chandra observatory on day 86 revealed a rich system of absorption lines superimposed on a supersoft source continuum without any emission lines. $\mathrm{H}, \mathrm{He}$, $\mathrm{C}$, and $\mathrm{N}$ absorption lines were blue-shifted by $1200 \mathrm{~km} / \mathrm{s}$. Modeling of the data with a blackbody indicates a photospheric temperature $\sim 310000 \mathrm{~K}$ [26]. The blue-shift $1200 \mathrm{~km} / \mathrm{s}$ of CV, CVI, NVI and NVII lines was confirmed on day 97 AM by a deep XMM-Newton observations [24]. According to [27] the last observation before the Swift satellite Sun constraint on 2014 January 6 (day $143 \mathrm{AM}$ ) yielded a rate $\sim 40$ count/s. The following observation by Swift on $2014 \mathrm{March}$ 4 showed the end of the SSS, because the count rate dropped to $\sim 0.8$ count/s (day $200 \mathrm{AM}$ ) and further to $\sim 0.4$ count/s on day $205 \mathrm{AM}$.

\subsection{Radio data}

The first detection of V339 Del in radio frequencies was done on 2013, Sep. 8 (day 22 AM) by VLA observatory in Soccoro at $28.2 \mathrm{GHz}$ (flux: 0.21 (3) mJy), $36.5 \mathrm{GHz}$ (flux 0.32(3) mJy) and CARMA in Inyo Mountains at 95.7 GHz [28]. On Sept. 25.9 (day $40 \mathrm{AM}$ ), nova was detected also at $15 \mathrm{GHz}$ (flux $0.68 \pm 0.18 \mathrm{mJy}$ ) by AMI near Cambridge [29]. According to Nelson [30], the ejecta were marginally resolved in May 2014 and appeared symmetric. They expanded very slowly with a rate of $\leq 0.07$ mas/day. Nelson suggested, that we see the object pole on.

\subsection{Infrared data}

Early infrared photometry in the JHKLM bands were published by many authors in ATels. Main results of the infrared photometry and spectroscopy can be found in [10],[31] and [32]. Analysis of the spectral energy distribution (SED) by [31] in a spectral range 0.36-5 $\mu \mathrm{m}$ has shown that the source near its optical brightness maximum mimics the emission of normal supergiant of spectral types B5 and A0 on Aug. 15.94 UT and Aug. 16.86 UT, respectively. The nova expansion velocity near its optical brightness maximum was $\sim 700 \mathrm{~km} / \mathrm{s}$. An infrared excess associated with the formation of a dust shell appeared in the SED one month after the nova brightness maximum. The dust component color temperature $1500 \mathrm{~K}$ was estimated from the $K-L$ color index on day 36 AM. The dust shell parameters were: radius $\sim 6.5 \times 10^{11} \mathrm{~m}$, luminosity $\sim 4 \times 10^{3} \mathrm{~L}_{\odot}$ and mass $\sim 1.6 \times 10^{21} \mathrm{~kg}$. The rate of dust supply to the nova shell was $\sim 8 \times 10^{-8} \mathrm{M}_{\odot} \mathrm{yr}^{-1}$. The expansion velocity of the dust shell was about $600 \mathrm{~km} / \mathrm{s}$. At IR maximum light (day $56 \mathrm{AM}$ ), the nova had a color index $J-L=6$, which corresponds to a color temperature $\sim 1100 \mathrm{~K}$ [10].

Gehrz et al. [32] derived a distance to the nova of $\sim 4.5 \mathrm{kpc}$ adopting an expansion velocity of the principal ejecta as $505 \mathrm{~km} / \mathrm{s}$, derived from the spectrum taken on day $25 \mathrm{AM}$. This distance gives an outburst luminosity of $\sim 8.3 \times 10^{-7} \mathrm{~L}_{\odot}$ (about 13 times the Eddington limit for one solar mass WD). The ejected gas mass as estimated by the cutoff wavelength during the free-free emission phase was $\sim 7.5 \times 10^{-5} \mathrm{M}_{\odot}$. On day $100 \mathrm{AM}$ the dust shell temperature was $\sim 850 \mathrm{~K}$ and its mass $\sim(1.2 \pm 0.4) \times 10^{-7} \mathrm{M}_{\odot}$. The dust consisted of amorphous carbon grains. The surprising fact that the dust formation occurred during the super-soft stage can be explained either by dense clumps in the ejecta or with a thick equatorial density enhancement. 


\subsection{Optical data}

\subsubsection{Photometry}

The $U B V R I$ photometric observations of the nova are available through AAVSO, VSNET and ATels. Photometric data in the first month of the outburst were gathered and analysed by [8]. Accurate and densely mapped $B V R_{C} I_{C}$ photometry in the first 77 days AM allowed Munari et al. [33] to find characteristic decline times from $V_{\max } \sim 4.46, B_{\max } \sim 4.70$ as $t_{2, V}=10.5, t_{3, V}=23.5$, $t_{2, B}=12, t_{3, B}=30$ days, that place V339 Del in a borderline position between fast and very fast novae according to classification scheme [34]. The light curve shows a smooth decline with a small plateau soon after maximum lasting 1.5 days and a longer plateau between the days 20 and 37 after maximum. The transition from optically thick to optically thin conditions of the ejecta occurred around the day $62 \mathrm{AM}$, when the nova declined in $V$ by 6 mag from its maximum brightness.

Highly accurate photometry obtained in the medium Stromgren $y, b$ bands and narrow [OIII], $\mathrm{H}_{\alpha}$ bands covering 500 days AM of the evolution of Nova Del 2013 was presented by Munari et al. [35]. These authors' comprehensive study reviews the behaviour of the nova in all regions of electromagnetic spectrum and provides unique information about extent and ionization of the ejecta, the onset of the critical phases like the transition between optically thick and thin conditions, and re-ionization by the central super-soft X-ray source. These authors explained very large variability in the super-soft X-ray emission (from day 69 to $86 \mathrm{AM}$ ) by clumpy ejecta passing through the line of sight.

Our $U B V R_{C} I_{C}$ CCD photometry of the nova was obtained by two $0.6 \mathrm{~m}$ telescopes and $60 / 180$ $\mathrm{mm}$ telephoto lens at the Stará Lesná Observatory (Slovakia), $0.5 \mathrm{~m}, 0.6 \mathrm{~m}$ and $1.25 \mathrm{~m}$ telescopes in Nauchny and $0.6 \mathrm{~m}$ and $1 \mathrm{~m}$ telescopes at Mt. Koshka in Crimea (Russia). The back-illuminated FLI ML3041 and Vers-Array 1300 CCD camera sensitive for $U$ band data were used at $0.6 \mathrm{~m}$ telescope in Stará Lesná and $1.25 \mathrm{~m}$ telescope in Nauchny, respectively. Some $U B V$ observations were obtained with photoelectric photometer at $0.6 \mathrm{~m}$ telescopes in Stará Lesná and Mt. Koshka. The data were processed in the usual way using the main standard star HD $194113(U=8.844(2)$, $\left.B=8.319(2), V=8.008(3), R_{C}=7.768(10), I_{C}=7.618(10)\right)$ and 15 check stars and reduced to the standard Johnson-Cousins system. Our observations were supplemented by all the available photometric data from AAVSO, VSNET, ATels and data from [33]. In Fig. 1 we present daily means of all data. Unusual behaviour of $V-R_{C}$ and $R_{C}-I_{C}$ color indices is mainly caused by an appearance and evolution of the strongest emission lines of [O III] $5007 \AA$ in $V$ band, $\mathrm{H}_{\alpha}$ in $R_{C}$ band and O I $7773 \AA$ and $8446 \AA$ in $I_{C}$ band. The evolution of emission lines published by [36] fully supports this explanation.

The track of the nova in the color-color diagram with dereddened indices $(U-B)_{0},(B-V)_{0}$ is shown in Fig. 2. V339 Del after maximum moved along the nova-giant sequence introduced by Hachisu and Kato [37]. This sequence is parallel to, but bluer in $(U-B) \approx-0.2 \mathrm{mag}$ than the supergiant sequence, because the mass of a nova envelope is 10000 times lower than that of a normal supergiant. After the nova reached the point of free-free emission, which coincides with the intersection of the blackbody and the nova-giant sequence, it evolved leftward, due to the development of strong emission lines.

Individual $U$ observations are plotted in Fig. 3. During the dust formation stage that started in the middle of September and lasted till December 2013, the $U$ light curve showed significant 


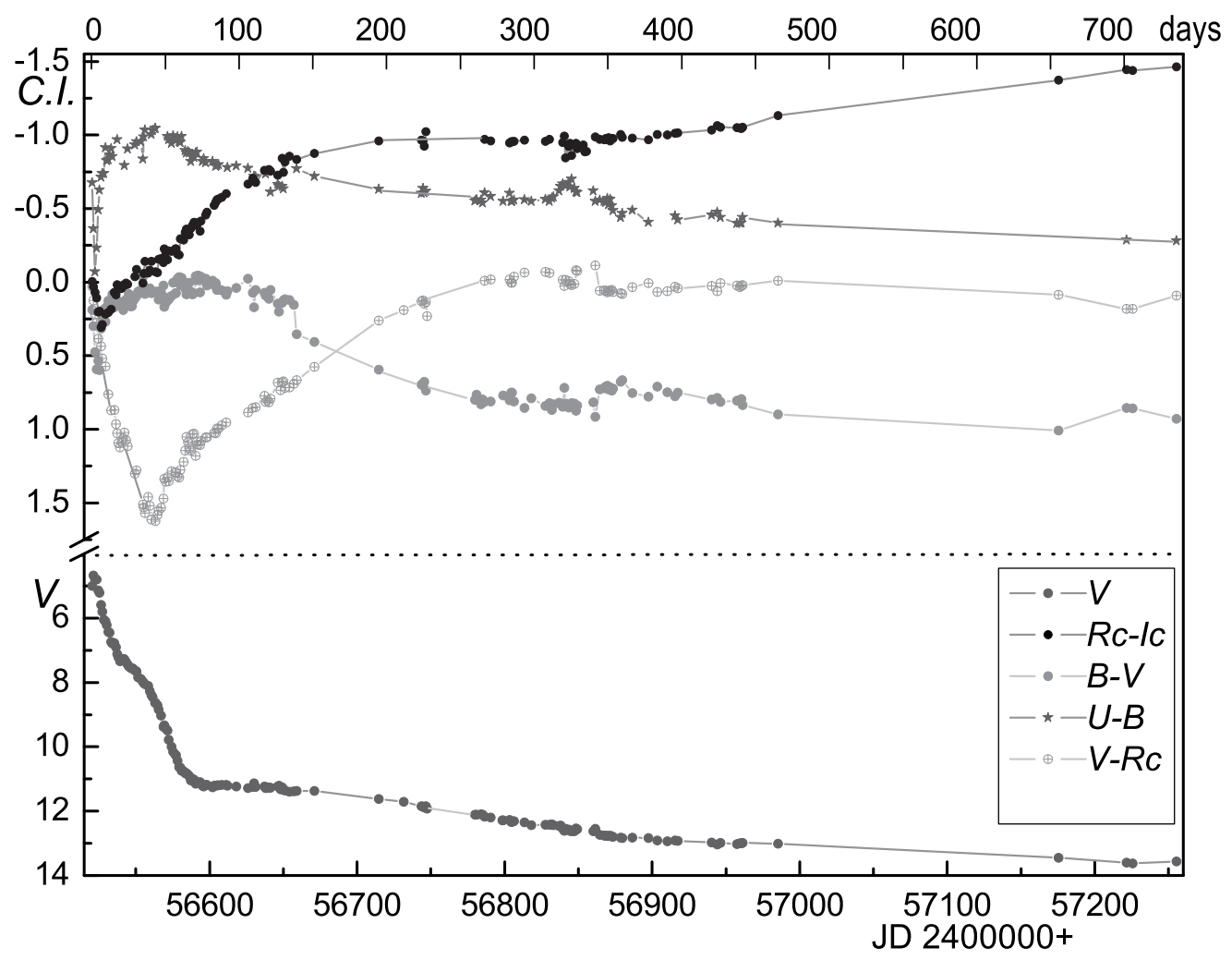

Figure 1: $V$ light curve and color indices curves of V339 Del.

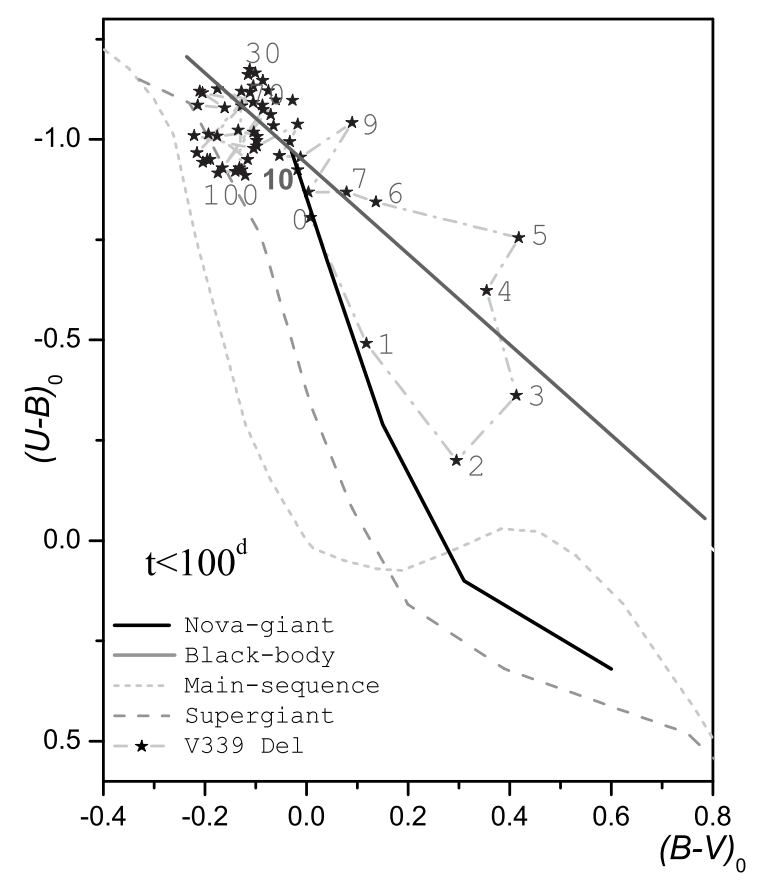

Figure 2: Track of the nova in $(U-B)_{0},(B-V)_{0}$ diagram, with dereddened data assuming $E(B-V)=$ 0.18 . 
variations detected also during night runs. The reason of these variations could be carbon dust grains formed in the dense clumps of matter in ejecta. The large amplitude variations of X-ray flux observed between the days 69 and 83 could be explained in the same way. We present also the brightness variations of the nova in two nights during the short-term increase of $U$ brightness in 2014. The graph is supplemented by the $B$ band brightness variations in 2015 .

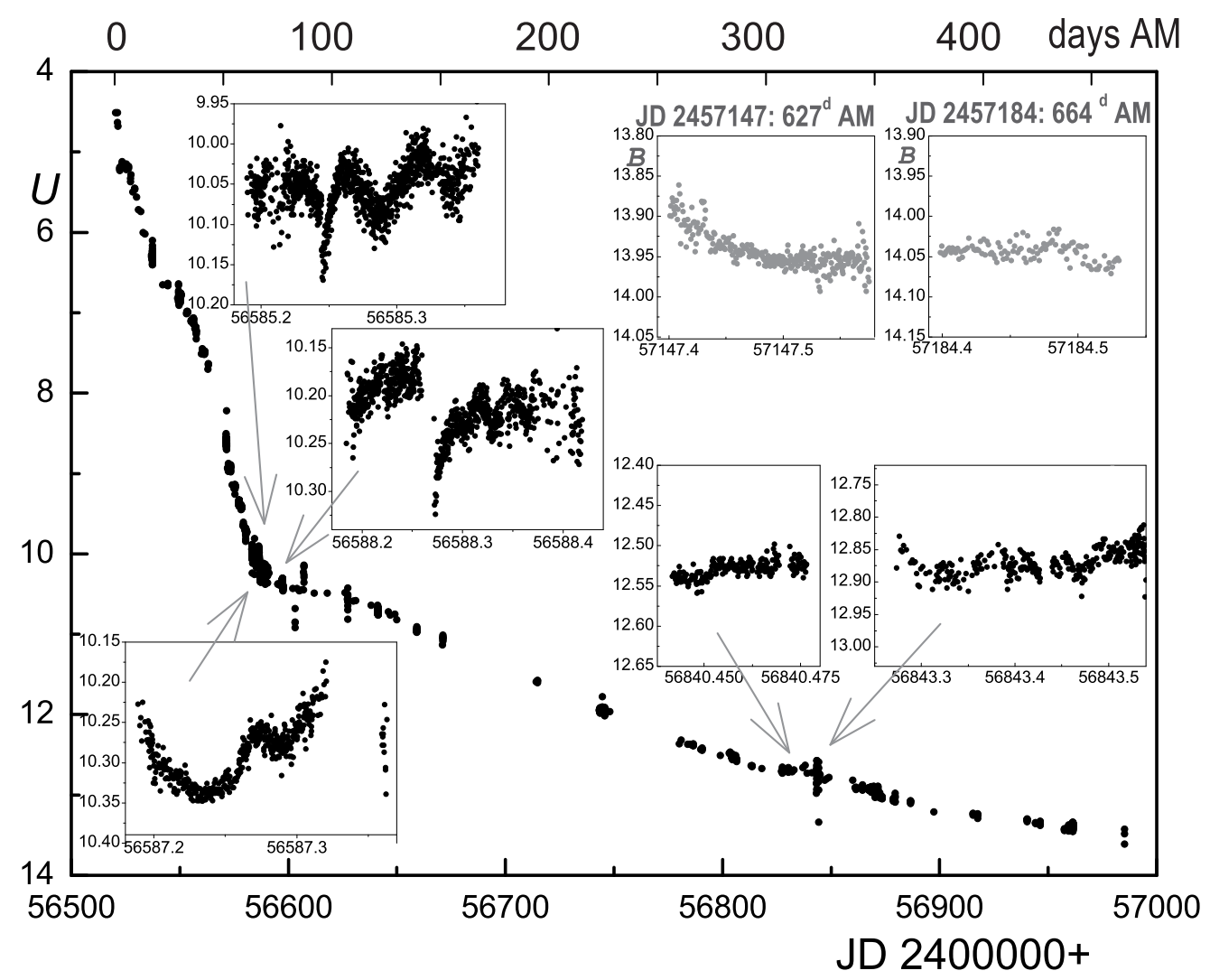

Figure 3: $U$ light curve and strong brightness variations on days 64, 66 and 67 AM. The monitoring was done in $U$ band on days 319 and 322 AM and $B$ band on days 627 and 664 AM.

Short-term $U$ light curve variability was caused by screening of the object by the dust clumps of the ejecta. In addition, there may have been effects related to the orbital motion. In the $U$ light curve taken between the days 50 to 76 AM (JD24456571-97) we removed the declining trend of the nova and searched for periods in residuals by Fourier period analysis. We found that the most likely period is 3.154 hours (Fig. 4), about half of the period of 6.43 hours found [8] in the $B, V$ data. The phase diagram in Fig. 5 was constructed using the ephemeris $\mathrm{HJD}_{\min }=2456575.397$ $+0.13140 \times \mathrm{E}$. The brightness variations probably reflect the orbital motion in the binary system V339 Del.

\subsubsection{Spectroscopy}

A large amount of spectroscopic observations of the nova were gathered by astronomers. The spectra taken during the first month of the outburst was described in [8]. The evolution of the nova was followed by amateurs of the ARAS group. They obtained and made publicly available 1151 


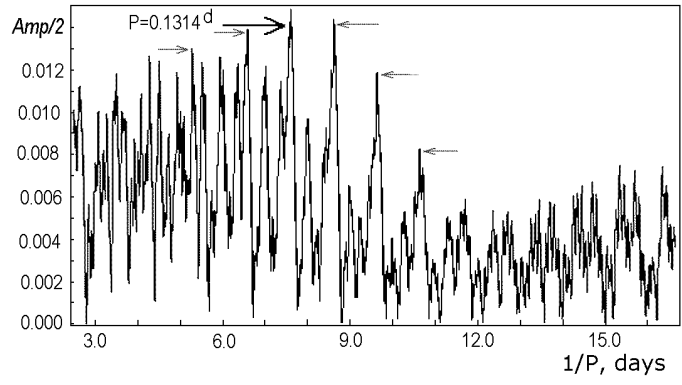

Figure 4: Fourier period analysis of $U$ data from day 50 to 76 AM (JD24456571 - 97).

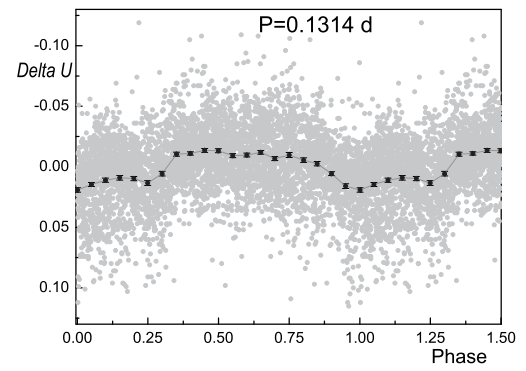

Figure 5: Phase diagram for the best period 3.154 hours.

spectra of V339 Del with spectral resolution ranging from 500 to 10000 during the time 2013 Aug 14 - 2015 Aug 17. The observations were described by Shore et al. [38], [39], [40] and used by Skopal et al. [41] to study the temporal evolution of the luminosity, radius and effective temperature of the nova shell from discovery to around day 40 . The above authors modelled the optical/near-IR spectral energy distribution (SED) using low-resolution spectroscopy (3500 - $9200 \AA$ ), $U B V R_{C} I_{C}$ and $J H K L M$ photometry and found that during the fireball stage (2013 Aug. $14.8-19.9), T_{\text {eff }}$ was in the range of $6000-12000 \mathrm{~K}$, the radius was expanding non-uniformly in time from $(\sim 66$ to $\sim 300)(d / 3 \mathrm{kpc}) \mathrm{R}_{\odot}$, while the luminosity was super-Eddington. The maximum of bolometric luminosity $\sim 9 \times 10^{38}(\mathrm{~d} / 3 \mathrm{kpc})^{2} \mathrm{erg} / \mathrm{s}$ occurred around Aug. 16.0, at maximum of $T_{\text {eff }}$, half a day before the visual maximum. The profile of the $\mathrm{H}_{\alpha}$ line and its relative high flux during the fireball phase suggest a low-density optically thin part of the envelope, which is ionized and has a bipolar shape. The optically thick/thin interface represents the warm expanding pseudo-photosphere. A gradual increase of the nebular contribution during the last day of the fireball stage (Aug. 18.9 19.9) may be caused by a gradual opening and enlargement of the H II zone and narrowing of the H I zone. The optically thick pseudo-photosphere was therefore transformed into a disk-like shape. After the fireball stage the mass of the ionized region was a few $\times 10^{-4} \mathrm{M}_{\odot}$ and mass loss rate between the days $5.5 \mathrm{AM}$ and $35 \mathrm{AM}$ decreased from $\sim 5.7$ to $\sim 0.71 \times 10^{-4} \mathrm{M}_{\odot}$. At the same time the temperature of the burning white dwarf increased from $\sim 37000 \mathrm{~K}$ to $\sim 150000 \mathrm{~K}$ and its radius decreased from 19 to $0.6 \mathrm{R}_{\odot}$. On day $35 \mathrm{AM}$ the model SED indicated a dust emission component in the spectrum. The dust was located beyond the $\mathrm{H} \mathrm{I}$ zone, where it was shielded from the radiation of the burning WD.

The emission feature around $6830 \AA$ observed in the spectra of nova from day 3 to 37 AM was identified by [41] as the Raman-scattered O VI $1032 \AA$ line because of similarity of its line profile with Raman $6825 \AA$ line profile in the spectrum of the symbiotic star Z And. Shore et al. [42] identified this emission as the C I $6828 \AA$ line, by comparing its profile with the profile of C I $8335 \AA$ line.

The observed galactic lithium abundance and theoretical predictions suggest that a significant amount of ${ }^{7} \mathrm{Li}$ could be produced in classical nova explosions. Tajitsu et al. [43] detected in high-resolution spectra of V339 Del taken 38-48 days after its explosion by $8.2 \mathrm{~m}$ Subaru telescope highly blue-shifted ( $\sim 1000 \mathrm{~km} / \mathrm{s})$ absorption resonance lines of singly ionized radioactive isotope of beryllium ${ }^{7} \mathrm{Be}$ (3130.583 and 3131.228 $\mathrm{A}$ ). Production of the unstable isotope ${ }^{7} \mathrm{Be}$ by the reaction ${ }^{3} \mathrm{He}(\alpha, \gamma){ }^{7} \mathrm{Be}$ in nova explosions was theoretically predicted by [44]. According to [45], 
${ }^{7} \mathrm{Be}$ decays to form ${ }^{7} \mathrm{Li}$ within a short time (half-life 53.22 days). Therefore, the detection of ${ }^{7} \mathrm{Be}$ in an expanding ejecta of the nova means, that it will soon decay to ${ }^{7} \mathrm{Li}$ on a time scale given by the half-life of ${ }^{7} \mathrm{Be}$. Hernanz et al. [46] showed that lithium production is favored for novae with underlying CO white dwarf. V339 Del is the first nova that has been observed to synthesize the element lithium.

The comprehensive high-resolution optical spectroscopy in the range 3800 and $8800 \AA$, taken by $1.2 \mathrm{~m}$ telescope TIGRE during the first 121 days of the outburst, was published by [47]. Their spectral line profiles atlas covers four different expansion phases of the ejecta. In optically thick phase H I, C I, Ca II, Fe II, O I exhibit clear P-Cyg profiles. Lowly ionized lines of Cr II, Fe I, Mg II, Na I, Sc II, Si II, Sr II, Ti II, show only narrow absorption features with radial velocities covering a narrow range between - 500 and $-800 \mathrm{~km} / \mathrm{s}$. H I P-Cyg profile show absorption with terminal velocity $-2000 \mathrm{~km} / \mathrm{s}$. During the transition phase that started on day 13 AM the spectrum exhibited strong H I lines with a weak P-Cyg absorptions that spans a velocity range from - 700 to - 1350 $\mathrm{km} / \mathrm{s}$. The terminal velocities of the strongest Fe II transitions were $-1200 \mathrm{~km} / \mathrm{s}$. The emission line profiles were asymmetric with the red side brighter than the blue one. The appearance of [N I] and [O I] lines marked the transition towards optically thin phase or nebular phase observed from day 40 AM. The P-Cyg absorptions disappeared. The ionization level of the gas increases as seen from appearance of He I lines and [O III] $5007 \AA$ line. The emission lines of H I, He I, C II, Fe II, O I and [O I] show double-peaked profile with separation of peaks $1400 \mathrm{~km} / \mathrm{s}$. It is clear signature of the non-spherical structure of the ejecta. During the bright X-ray central source phase covered by observations from day $70 \mathrm{AM}$ the appearance of high ionization N III, O III, He II and [Fe VII] emission lines suggests re-ionization of expanding ejecta by highly energetic radiation from the remnant white dwarf.

The medium-resolution spectrum of V339 Del that we obtained in August 2015 with the SCORPIO spectrograph of the $6 \mathrm{~m}$ telescope in North Caucasus is shown in Fig. 6. Double-peaked profile of emission lines confirms a non-spherical structure of the ejecting shell.

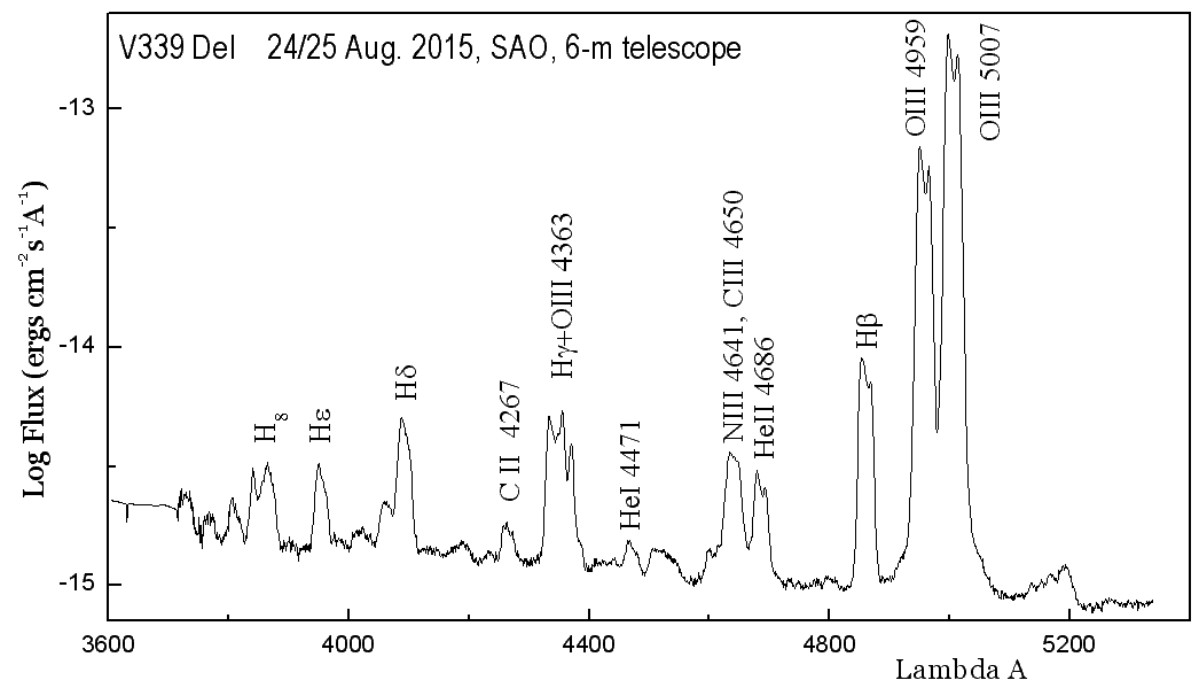

Figure 6: Medium resolution spectrum of V339 Del.

Acknowledgements. We acknowledge with thanks the variable star observations from the 
AAVSO International Database contributed by observers worldwide and used in this research. This study was supported by the VEGA grants $2 / 0143 / 14,2 / 0002 / 13$, Russian president grant NSh9670.2016.2 and RFBR grants No. 15-02-06178 and 11-02-01213a. Observations with the 6-m BTA telescope are conducted with the financial support of the Ministry of education and science of the Russian Federation (agreement No. 14.619.21.0004, project ID RFMEFI61914X0004).

\section{References}

[1] R.A. Downes and H.W. Duerbeck, Optical imaging of nova shells and the maximum magnitude of decline relationship, 2000 AJ 1202007

[2] K. Itagaki, V339 Delphini = Nova Delphini 2013 = PNV J20233073+2046041, 2013 IAUC 9258

[3] S. Samus, V339 Delphini = Nova Delphini $2013=$ PNV J20233073+2046041, 2013 IAUC 9258

[4] M.J. Darnley, M.F. Bode, R.J. Smith and A. Evans, Spectroscopic Observation of PNV J20233073+2046041 with the Liverpool Telescope, 2013 ATel 5279

[5] S.N. Shore, P. Skoda and P. Rutsch, Further spectroscopic observations of Nova Del $2013=P N V$ J20233073+2046041 with the Ondrejov Observatory, 2013 ATel 5282

[6] U. Munari, P. Valisa, A. Milani and G. Cetrulo, Spectroscopy of the very fast Nova Del 2013, already declining past maximum brightness, 2013 ATel 5297

[7] U. Munari and T. Zwitter, Equivalent width of Na I and K I lines and reddening, 1997 Astron. Astrophys. 318269

[8] D. Chochol, S. Shugarov, T. Pribulla and I. Volkov, Photometry and spectroscopy of the classical nova V339 Del (Nova Del 2013) in the first month after outburst, 2014 CoSka 43330

[9] M. Livio, Classical novae and the extragalactic distance scale, 1992 ApJ 393516

[10] M.A. Burlak et al. UBVJHKLM photometry and low-resolution spectroscopy of Nova Delphini 2013 (V339 Del), 2015 Baltic Astronomy 24109

[11] G.H. Schaefer et al., The expanding fireball of Nova Delphini 2013, 2014 Nature 515234

[12] S.N. Shore, Correction: First high resolution ultraviolet (HST/STIS) and supporting optical spectroscopy of V339 Del = Nova Del 2013, 2013 ATel 5410

[13] D. Denisenko, V339 Delphini = Nova Delphini $2013=$ PNV J20233073+2046041, 2013 IAUC 9258

[14] U. Munari and A. Henden, Photometry of the progenitor of Nova Del 2013 (V339 Del) and calibration of a deep BVRI photometric comparison sequence, 2013 IBVS 6087

[15] N.R. Deacon et al., Pre-outburst observations of Nova Del 2013 from Pan-STARRS 1, 2014 Astron. Astrophys. 563 A129

[16] M. Ackermann and The Fermi-LAT Collaboration, Fermi establishes classical novae as a distinct class of gamma-ray sources, 2014 Science 345554

[17] C.C. Cheung, P. Jean, S.N. Shore and The Fermi-LAT Collaboration, Fermi-LAT gamma-ray observations of Nova Centauri 2013, 2013 ATel 5649

[18] B.D. Metzger et al., Gamma-ray novae as probes of relativistic particle acceleration at non-relativistic shocks, 2015 MNRAS 4502739 
[19] L. Chomiuk et al., Binary orbits as the driver of $\gamma$-ray emission and mass ejection in classical novae, 2014 Nature 514339

[20] K.L. Page and A.P. Beardmore, V339 Del (Nova Del 2013) is a weak non-super-soft X-ray source, 2013 ATel 5429

[21] K.L. Page et al., Detection of super-soft emission in nova V339 Del, 2013 ATel 5470

[22] J.P. Osborne et al., Nova Del 2013 (V339 Del) is now a bright super-soft X-ray source, 2013 ATel 5505

[23] A.P. Beardmore, J.P. Osborne and K.L. Page, Large amplitude super-soft X-ray intensity variations and a 54 sec QPO in Nova Del 2013 (V339 Del), 2013 ATel 5573

[24] J.U. Ness et al., Confirmation of the Swift 54-second period and detection of a strong C V absorption edge in a deep XMM-Newton observation of V339 Del, 2013 ATel 5626

[25] J.U. Ness et al., Short-period X-ray oscillations in super-soft novae and persistent super-soft sources, 2015 Astron. Astrophys. 57839

[26] T. Nelson, K. Mukai, L. Chomiuk and J. Sokoloski, Chandra observations reveal a rich absorption line system in the supersoft X-ray spectrum of V339 Del (Nova Del 2013), 2013 ATel 5593

[27] K.L. Page et al., Swift observations of the fading of V339 Del, 2014 ATel 5967

[28] L. Chomiuk et al., Radio/Millimeter detection of Nova V339 Del, 2013 ATel 5382

[29] G. Anderson, R. Fender, L. Chomiuk and T. Staley, AMI detection of Nova V339 Del, 2013 ATel 5428

[30] T. Nelson, Multiwavelength observations of mass ejection in novae, 2014 [http://fermi.gsfc.nasa.gov/science/mtgs/symposia/2014/program/05Nelson.pdf]

[31] O.G. Taranova, A.M. Tatarnikov, V.I. Shenavrin and A.A. Tatarnikova, Infrared photometry of Nova Delphini 2013 (=V339 Del) in the first sixty days after its outburst, 2014 Astronomy Letters 40120

[32] R.D. Gehrz et al., The early infrared temporal development of Nova Delphini 2013 (V339 DEL) observed with the Stratospheric Observatory for Infrared Astronomy (SOFIA) and from the ground, 2015 ApJ 812132

[33] U. Munari, A. Henden, S. Dallaporta and G. Cherini, Photometric evolution of Nova Del 2013 (V339 Del) during the optically thick phase, 2013 IBVS 6080

[34] B. Warner, Cataclysmic variable stars, 1995 Cambridge Astrophys. Ser., vol. 28

[35] U. Munari, A. Maitan, S. Moretti and S. Tomaselli, 500 days of Stromgren b,y and narrow-band

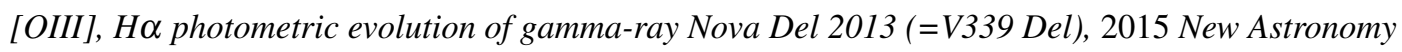
4028

[36] H.D. Moers and W.S. Wiethoff, 125-day spectral record of the bright Nova Delphini 2013 (V339 Del), 2014 JAAVSO 42161

[37] I. Hachisu and M. Kato, The UBV color evolution of classical novae. I. Nova-giant sequence in the color-color diagram, 2014 ApJ 78597

[38] S.N. Shore et al., Continuing spectroscopic observations (3500-8800A) of Nova Del 2013 with the Ondrejov Observatory and the ARAS group, 2013 ATel 5312

[39] S.N. Shore et al., Continuing optical spectroscopy of V339 Del = Nova Del 2013 with the Nordic Optical Telescope and the ARAS group, 2013 ATel 5378 
[40] S.N. Shore et al., Continuing spectroscopic observations (3600-8800A) of V339 Del = Nova Del 2013 in the early nebular stage with the Nordic Optical Telescope, Ondrejov Observatory and the ARAS group, 2013 ATel 5546

[41] A. Skopal et al., Early evolution of the extraordinary Nova Delphini 2013 (V339 Del), 2014 Astron. Astrophys. 569 A112

[42] S.N.Shore, I. De Gennaro Aquino, S. Scaringi and H. van Winckel, On the Raman O VI and related lines in classical novae, 2014 Astron. Astrophys. 570 L4

[43] A. Tajitsu et al., Explosive lithium production in the classical nova V339 Del (Nova Delphini 2013), 2015 Nature $\mathbf{5 1 8} 381$

[44] M. Arnould and H. Norgard, The Explosive Thermonuclear Formation of ${ }^{7}$ Li and ${ }^{11}$ B, 1975 Astron. Astrophys. 4255

[45] G. Audi, O. Bersillon, J. Blachot and A.H. Wapstra, The NUBASE evaluation of nuclear and decay properties, 2003 Nucl. Phys. A 7293

[46] M. Hernanz, J. Jose, A. and J. Isern, On the synthesis of ${ }^{7} \mathrm{Li}$ and ${ }^{7}$ Be in novae, 1996 ApJ 465 L27

[47] I. De Gennaro Aquino et al., High spectral resolution monitoring of Nova V339 Delphini with TIGRE, 2015 Astron. Astrophys. 581 A134

[48] C. de Vegt, Nova Cygni 1975, 1975 IAUC 2826

\section{DISCUSSION}

VOJTECH ŠIMON: You showed also pre-outburst phase of the optical activity of V339 Del. Can you please compare the optical brightness in the phases before and after the outburst of V339 Del.

DRAHOMÍR CHOCHOL: The brightness of the nova in quiescence was $B \sim 17.2$ mag. At present the nova is still in outburst, because we found $B=14.3$ mag from our last photometric observations obtained on 2015 August 20. 